\title{
CONTROL TEST OF A WEARABLE ELBOW ORTHOSIS WITH BI-MUSCULAR PNEUMATIC SERVO-DRIVE BASED ON BRAIN-COMPUTER INTERFACE
}

\author{
R. Dindorf*, P. Wos ${ }^{* *}$
}

\begin{abstract}
The paper presents a control tests of a wearable orthosis of elbow joint with a bi-muscular pneumatic servo-drive. Brain-computer interface (BCI) based control is a complex task, because it must contain a device for recording bioelectric signals (BESs) and translate them into control signals (CS) of the elbow orthosis. The authors analysed the impact of the induced brain activity and the muscular tension within the head of the participant on the BESs which can be used to control the pneumatic servo drive of the elbow joint orthosis. For controlling the elbow joint orthosis, a distributed control system (DCS) was developed that contains two control layers: a master layer connected to the BCI device and a direct layer contained in a wireless manner with the controller of the pneumatic servo-drive (PSD). In the programming of the PSD controller, a kinematic-dynamic model of the elbow joint orthosis, patterned after the biological model of human biceps-triceps, was used. A biomimetic dynamic model of the pneumatic muscle actuator (PMA) was used, in which the contraction force results from the adopted exponential static model of the pneumatic muscle (PM). Use of direct visual feedback (DVF) makes it possible for the participant to focus on the movement of the orthosis taking into account the motoric functions of the elbow.
\end{abstract}

Keywords: Pneumatic muscle servo-drive, Wearable elbow orthosis, Wireless interface controller, Brain computer interface

\section{Introduction}

Progress in the fields of biomechanics and robotics has made it possible to create a completely new group of biomechanical equipment, which includes wearable orthoses with drives. Those orthoses are intended to support or replace the functions of the muscular system of human extremities. Traditional orthoses use electric drives which, due to their high rigidity, can cause hazards to human joints. Because human muscles are flexible and responsive; therefore, drives of orthoses must be made using pneumatic muscle actuators (PMAs) that are analogous to human skeletal muscles (Vetrice et.al., 2016). The PMAs performing smooth movements and are free from the slip-stick phenomenon and have natural damping properties (Dindorf et.al., 2017). As a result of the flexible properties of PMAs, they are often used to drive elbow orthosis (Ripel et.al., 2014). In order to ensure safe use of an active orthosis with a pneumatic muscle servo-drive, the user must supervise its movement range and load.

\section{Test stand}

Tests of control of the elbow joint orthosis with a bi-muscular pneumatic servo-drive were performed based on a brain-computer interface (BCI). The BCI device with surface electrodes (SEs) transforms bioelectric signals (BESs) induced by brain activity and muscle tension into control signals (CSs) that can be used to servo control the elbow joint orthosis. Direct visual observation of the movement of an elbow

Prof. Ryszard Dindorf: Kielce University of Technology, al.Tysiaclecia Panstwa Polskiego 7, 25-314 Kielce, PL, dindorf@tu.kielce.pl

** EngD. Piotr Wos: Kielce University of Technology, al.Tysiaclecia Panstwa Polskiego 7, 25-314 Kielce, PL, wos@tu.kielce.pl 
joint orthosis by the participant is referred to as direct visual feedback (DVF). The general model of control of a wearable elbow joint orthosis based on a brain-computer interface (BCI) and direct visual feedback (DVF) are shown in Figure 1. A block diagram of the elbow joint orthosis control system is shown in Figure 2.

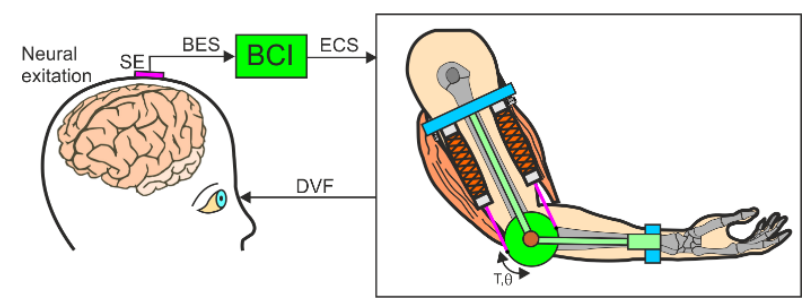

Fig. 1. General control model of the wearable elbow orthosis

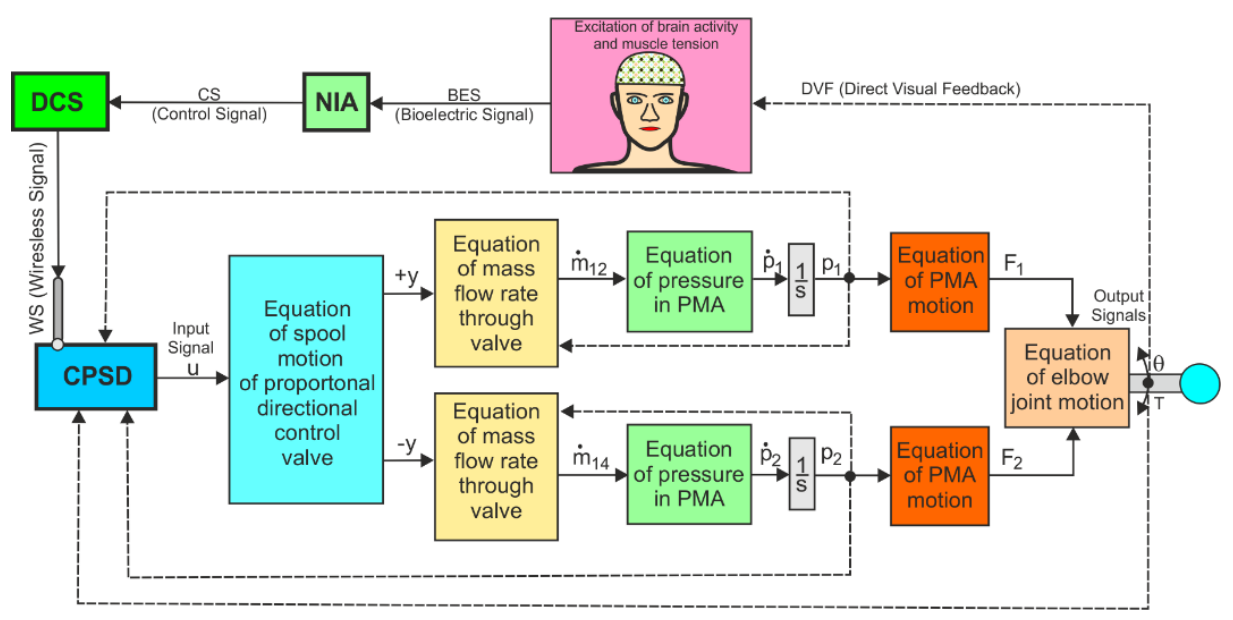

Fig. 2. Block diagram of the elbow orthosis control system

The block diagram of the control of the elbow orthosis on the test stand and the view of the elbow orthosis put on the participant's arm are shown in Figure 3. The orthosis is fastened with straps on the sides of the participant's forearm and arm. The weight of the mechanical parts of the orthosis should be distributed on the entire upper limb.

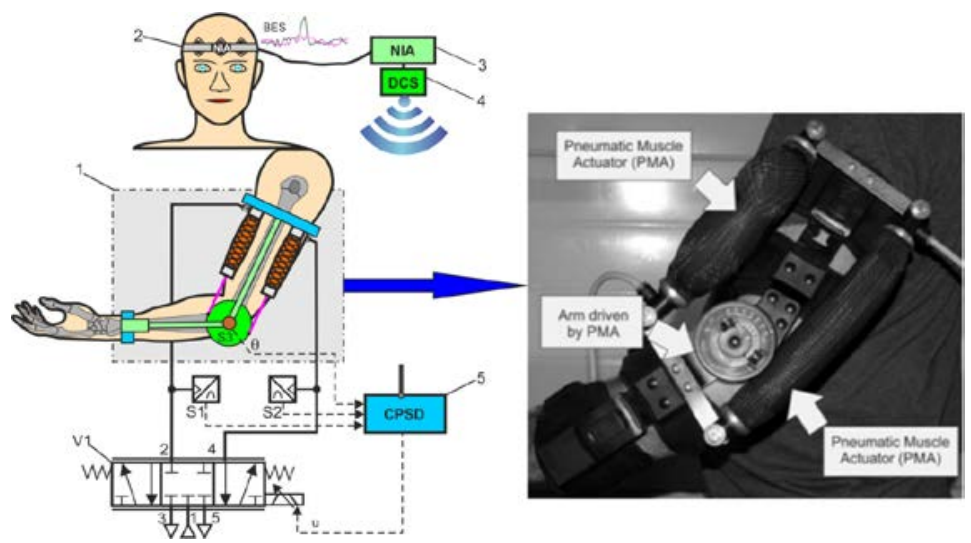

Fig. 3. Diagram of the test stand: 1 - wearable elbow orthosis with bi-muscular pneumatic servo-drive, 2 - headband, 3 - Neural Impulse Actuator (NIA), 4 - Distributed Control Systems (DCS), 6 - Controller of Pneumatic Servo-Drive (CPSD), V1 - proportional directional control valve, S1,S2 pressure transducers, S3 - rotary encoder

\section{Control test}

Once the NIA has captured BESs they are analyzed and separated through fast Fourier transforms (FFT) into different frequencies in order to be translated into commands that the user defines as control signals to execute orthosis movement with a bi-muscular pneumatic servo-drive. For the purpose of control of bending/straightening of the orthosis, control commands were selected, $u=(u p$, down). During the detection, recording and decoding of the BESs to ECSs, there are two main problems. The first problem is 
the signal to noise ratio. The second problem is the noise signal defined as an electrical signal that is not part of the desired BES. The measured BES can have both positive and negative voltage (Fig. 4a). Another issue is signal interference, which means that the relative contribution of any frequency in the BES should be not changed (Fig. 4b).
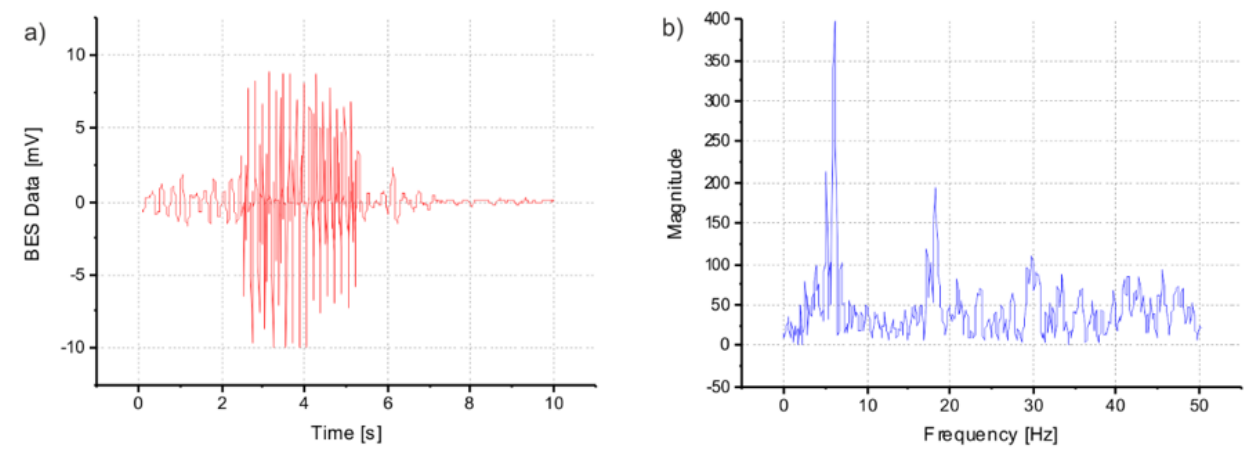

Fig. 4. Generating characteristics of a single channel signal (a) using a frequency analysis (b)

The measured BESs can be processed to eliminate noise or other possible interference. Consequently, the signal is often straightened and averaged. Initial biosignal processing usually involves signal filtration and other methods of removing noise and interference (e.g. physiological artefacts). At this stage, digital filters, spatial filters and signal whitening methods are used. These different approaches have been considered and developed to perform the analysis in the frequency domain and to compute power spectral density (PSD) of the bioelectric signals (BESs). Research on the brain-computer interface shows that the distinction of mental tasks can be carried out selectively in selected frequency bands. Features obtained by means of discrete Fourier transform (DFT) illustrate the amplitude of the signal for individual frequency components. The DFT can be written as follows (Pampu, 2011):

$$
X_{i}(k)=\sum_{n=0}^{M-1} X_{i}(n) w(n) e^{-j \omega_{k} n} \quad 1 \leq i \leq K
$$

where: $x_{i}$ - measured BES as the input variables. $n$ - number of the signal sample, $M$ - samples per segment, $K-$ smaller segment, $w(n)$ - windows, $\omega_{k}$ - discrete frequency of DFT, $\omega_{k}=2 \pi k / \mathrm{N}, k-$ frequency bins, $N$ - number of samples, $N=M R, R$ - number of non-overlapping frames.

From (1) can be obtained the power of BES as:

$$
P_{i}(k)=\frac{1}{M U}\left[X_{i}(k)\right]^{2}
$$

where the normalisation factor $U$ is defined:

$$
U=\frac{1}{M} \sum_{n=0}^{M-1} w^{2}(n)
$$

Finally, the power of BES in a frequency band can be calculated as follows:

$$
P_{X X}(k)=\frac{1}{K} \sum_{i=1}^{K-1} P_{i}(k)
$$

For real signals, due to the symmetry of the spectrum, it is sufficient to account for only half of the N/2 stripes. It is known that the useful biosignal band is in the range from $0.5 \mathrm{~Hz}$ to $50 \mathrm{~Hz}$. Due to the overall power spectrum, it is possible to identify a disturbance of the bio-potential baseline and distinguish it from increased brain activity. In order to convert the BES to a useful control signal, all negative amplitudes are firstly converted into positive amplitudes. Negative peaks are transferred into the positive direction. In addition to the transparency of the record, the aim of this action is to obtain the ability to plot curves for standard amplitude parameters, such as mean, peak - maximum and field values (the raw BES record has an average amplitude value equal to zero). To solve this problem, a unique part of the signal is minimised by using digital smoothing algorithms that emphasise the main direction in which the biosignal travels (Fig. 5). Peaks with excessive amplitude are trimmed; the signal is linearised. Three algorithms are used here: application of the FFT filter, the low-pass filter and the Lowess method (LM) weighted local 
polynomial regression fitting. As a result of the experiment, it was concluded that BES caused by muscle tension in the participant's head, e.g. pressing the tongue against the palate or clenching of jaws with bigger or smaller force, provides the best effects of generation of control signals to the bi-muscular pneumatic servo-drive of elbow orthosis.

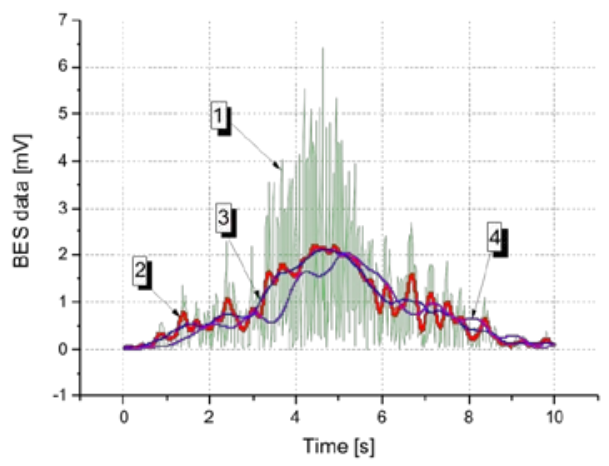

Fig. 5. Processing for smooth BES data: 1 - BES data (absolute valve), 2 - FFT filter, 3 - Lowess method (LM), 4 - Low-pass filter (15 Hz cut off)

The precise movement control of the elbow orthosis can be increased by a participant undergoing appropriate training. Figure 6 shows an example of a result of a follow-up control of the bending angle of the elbow joint orthosis using bioelectric signals. The analysis of the graphs shows that there is a delay between the value of the set signal and the angle of bending of the orthosis. This delay is not important as the participant controls the elbow orthosis with direct visual feedback (DVF).

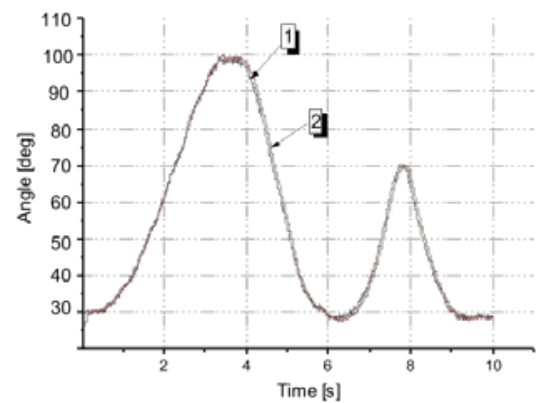

Fig 6. Follow-up control results of elbow orthosis: 1 - setpoint angle, 2 - current angle

\section{Conclusions}

As a result of the experiment, it was concluded that BES with frequencies of up to $40 \mathrm{~Hz}$ and amplitudes of several $\mathrm{mV}$, caused by muscle tension in the participant's head, e.g. pressing the tongue against the palate or clenching of jaws with bigger or smaller force, provides the best effects of generation of control signals to the bi-muscular pneumatic servo-drive of elbow orthosis. The advantage of the direct visual feedback (DVF) is the user's concentration on the forced movement of the orthosis associated with the motoric functions of the elbow. Such feedback ensures safe use of the brain-controlled elbow orthosis with a pneumatic muscle servo-drive. Precise control of the elbow joint orthosis in accordance with the user's expectations depends on its preparation (training). Training of the participant includes teaching him or her how to generate specific BESs that can be used to control the elbow orthosis.

\section{References}

Dindorf, R., Takosoglu, J. and Woś, P. (2017) Developments of pneumatic control systems. Monograph M89. Kielce University of Technology, Kielce (in Poland).

Pampu, N.C. (2011) Study of effects of the short time fourier transform configuration on EEG spectral estimates. Acta Technica Napocensis, Electronics and Telecommunications, 4, pp.26-29.

Ripel, T., Krejsa, J., Hrbacek, J. and Cizmar, I. (2014) Active Elbow Orthosis. International Journal of Advanced Robotic Systems, 1, pp.1-10.

Vetrice, G. and Deaconescu, A. (2016) Elbow joint rehabilitation equipment actuated by pneumatic muscles. In: Proceedings of the $4^{\text {th }}$ International Conference on Computing and Solutions in Manufacturing Engineering (CoSME'16), 4 January 2016, pp.1-6. 